\title{
Effect of morphine withdrawal on activity of gaba in the brain of rats
}

\begin{abstract}
The aim of the study was to explain whether the activities of the GABA-catabolizing enzymes (succinate dehydrogenase (SDH), SSA-dehydrogenase (SSA-DH) and GABAtransaminase (GABA-T) altered in cerebellum and thalamus of rats in the different terms after morphine withdrawal (MW). The 24hours' morphine withdrawal caused reliable changes in the enzymes activity only in thalamus. After 3 and 7day's morphine withdrawal the activation of SDH and GABA-shunt was determined in both the brain regions tested. Possible explanation of the changes observed may be related to contradaptational events recalled by chronic morphine intoxication in the GABAergic neurons.
\end{abstract}

Keywords: GABA, syndrome; VTA, cerebellum; VLO, enzymes, morphine
Volume 2 Issue 5 - 2015

\author{
Husam Abazid \\ Department of Medical Biochemistry and Microbiology, Isra \\ University, Jordan
}

Correspondence: Husam Abazid, Department of Biochemistry and Microbiology, Isra University, Faculty of Pharmacy, Amman, Jordan,Email husambazid@yahoo.com

Received: October 21, 2015 | Published: November 17, 2015
Abbreviations: SDH, succinate dehydrogenase; SSA-DH, ssa-dehydrogenase; GABA-T, GABA-transaminase; VTA, ventral tegmental area; VLO, ventrolateral orbital cortex; MW, morphine withdrawal

\section{Introduction}

In the formation of opiate addiction disorders play significant role interaction neurotransmitter systems. ${ }^{1-4}$ This concern practically all studied to date neurotransmitters: dopamine, nor epinephrine, serotonin, glutamic acid, g-aminobutyric acid (GABA), etc. Shifts in the neurotransmission systems are directly related to the formation of the phenomenon of addiction, withdrawal syndrome and tolerance..$^{2,4}$ It has been shown that the administration of morphine in rats leads to the activation of the dopaminergic in the ventral tegmental area and the nucleus accumbens (reward system centers) due to inhibition of GABAergic neurons. ${ }^{2}$ Opioids are the most potent analgesics in clinical use; however, their powerful rewarding properties can lead to addiction.

The scientific challenge is to retain analgesic potency while limiting the development of tolerance, dependence, and addiction. Both rewarding and analgesic actions of opioids depend upon actions at the mu opioid (MOP) receptor. Systemic opioid reward requires MOP receptor function in the midbrain ventral tegmental area (VTA) which contains dopaminergic neurons. ${ }^{4}$ These biochemical and pharmacological studies indicate participation of the GABA system in a number of effects of morphine, the formation of tolerance and dependence on the drug. ${ }^{5} \mathrm{~A}$ decrease in delta receptor positive cell density was observed in the CA1, CA3 and dentate gyrus without alteration of the distribution across the different GABAergic populations that mainly express delta receptors. ${ }^{6}$

In the brain, about $17 \%$ a-ketoglutarate is converted to succinate by the enzymes (GABA metabolism), forming so-called GABA shunt. Ratio reactions GABA shunt, and Krebs cycle differently in brain and depends on the concentration of GABA-ergic paths. ${ }^{7,8}$ It has been suggested that activation of GABA-metabolizing enzymes may help to remedy any deficiency of energy substrates in certain pathological conditions involving inhibition of energy metabolism in neurons (ischemia, hypoxia, etc.). ${ }^{9}$ Based on the above, the aim of this investigation was to study the state of GABA metabolism and succinate dehydrogenase activity in some brain regions of rats characterized by a high content of GABAergic neurons (cerebellum, thalamus) the abolition of morphine in chronic morphine intoxication. Opioid addiction is a major medical and social problem worldwide. Chronic use of opioids causes long-term adaptations in the nervous system.

These neuroadaptations underlie behavioral manifestations of opioid dependence and are thought to be important in the compulsive nature of drug taking, drug craving, and vulnerability to relapse during abstinence. In this article, we discuss the consequences of opioid use in a chronological manner from first drug encounters to addiction and relapse. ${ }^{10}$ Presynaptic effect of mu-opioid receptor activation on the GABAergic neurons in the ventrolateral orbital cortex (VLO). The current data suggests that a presynaptic inhibition of the GABA release may contribute to the mu-opioid receptor mediated effects in the VLO and provides novel electrophysiological evidence for the underlying mechanisms of mu-opioid. ${ }^{11}$

\section{Materials and methods}

Experiments performed on white mongrel male rats weighing $180-200 \mathrm{~g}$. Animals were divided into four groups, eight animals in each. Control animals received simultaneously an equivalent of $0.9 \%$ sodium chloride solution (I group). Chronic morphine intoxication induced by intraperitoneal injection of $1 \%$ solution of morphine hydrochloride during 7 days. The first two days the drug was given at $10 \mathrm{mg} / \mathrm{kg}$ body weight twice a day, 3-4days $-15 \mathrm{mg} / \mathrm{kg}$ twice a day, 5-7days $-20 \mathrm{mg} / \mathrm{kg}$ body weight twice a day. The animals were decapitated after 24hours (II group), 3days (III group) and 7days (IV group) after the last injection of morphine. Brains were divided into sections by Glowinski, Iversen, 1974, cerebellum and thalamus was isolated and immediately frozen in liquid nitrogen. The parts of the brain homogenates was determined by the activity of metabolic enzymes GABA: GABA-transaminase (GABA-T), and succinic semialdehyde dehydrogenase (SSA-DH), ${ }^{12}$ as well as - of succinate dehydrogenase $(\mathrm{SDH}){ }^{13}$ Protein was determined by Lowry. The significance of differences between groups was evaluated using a parametric method Student's t-test.

\section{Results and discussion}

Administration of morphine to rats for 7-14days leads to formation of physical dependence syndrome and drug injection termination causes morphine withdrawal symptoms (MWS). ${ }^{14}$ However, none of the experimental groups, we observed no overt signs of CNS 
hyper excitability. The degree of adaptation shifts caused by chronic administration of morphine was assessed by changes in the studied parameters GABA metabolism and the citric acid cycle. 24hours after the last administration of morphine (II group) in the thalamic region noted increased activity of (SSA-DH) $27.0 \%$ due to lower activity of SDH $(25 \%)$. In the cerebellum did not observe significant changes in the activity of the enzymes studied (Table 1).

Table I The activity of metabolic enzymes GABA and SDH in the brain of rats under morphine withdrawal syndrome (MWS) (in nmol/min/mg protein)

\begin{tabular}{|c|c|c|c|c|}
\hline Groups Indicators & I. Control & II. MWS-24 hours & III.WS - 3 days & IV. MWS-7 days \\
\hline & Cerebellum & & & \\
\hline GABA-T & $1,82 \pm 0,16$ & $1,75 \pm 0,08$ & $3,17 \pm 0,19 *$ & $2,58 \pm 0,14 *$ \\
\hline SSA-DH & $12,97 \pm 0,55$ & $13,38 \pm 0,53$ & $16,56 \pm 0,37 *$ & $11,42 \pm 0,62$ \\
\hline \multirow[t]{2}{*}{$\mathrm{SDH}$} & $37,90 \pm 1,28$ & $29,90 \pm I, 4 I$ & $64,93 \pm 1,19 *$ & $54,92 \pm 1,15 *$ \\
\hline & Thalamus & & & \\
\hline GABA-T & $1,60 \pm 0,10$ & $1,74 \pm 0,05$ & $1,96 \pm 0,10 *$ & $2,02 \pm 0,09 *$ \\
\hline SSA-DH & $12,17 \pm 0,36$ & $15,45 \pm 0,37 *$ & $17,34 \pm 0,45 *$ & $8,53 \pm 0,40 *$ \\
\hline $\mathrm{SDH}$ & $32,92 \pm 1,07$ & $24,64 \pm 0,53 *$ & $49,47 \pm 1,02 *$ & $48,39 \pm 1,42 *$ \\
\hline
\end{tabular}

* - significant changes in relation to the control group.

By increasing the morphine withdrawal period up to 3 days in both brain regions was noted significant activation of the enzyme GABAshunt on the background of increasing the activity of succinate dehydrogenase. After 7days, the MWS in the cerebellum and thalamus remained increased activity of GABA-T and SDH. Activity SSA-DH in the IV group has not changed significantly in the cerebellum, and was lower than the control in the thalamic region (Table 1). According to the concepts of "allostasis", the development of drug addiction at the molecular level, accompanied by an increase adaptive changes in neurons within a certain level of non-normal (homeostasis).

The formation of such allostatic changes during addiction supposedly involved a number of anatomical structures of the central nervous system connected to the so-called cortico-striatalthalamic loop. ${ }^{15}$ According to others, prolonged administration of opioids leads to a stabilization of the activity of dopamine and nor epinephrine-ergic systems with some reduced level of activity on the background of oppression GABA-ergic inhibitory neurons. ${ }^{2,3}$ In relation to this background, the abolition of receipt of morphine in the body causes the activation of excitatory neurotransmitter systems while maintaining decreased inhibitory control. The most pronounced activation of GABA-catabolizes enzymes and SDH were observed in long periods of morphine withdrawal (III and IV) in both studied brain structures see Table 1. In group II, significant changes in the activity of the enzymes occurred only in the thalamic region. According to the literature in the thalamic area contains a high concentration of opiate receptors that are virtually not found in the cerebellum. ${ }^{16}$

However, in both studied brain structures we observed unidirectional changes in the activity of the enzyme GABA-shunt when abolition of morphine. We can assume that the observed effect is a manifestation of a general Rear adaptive process caused by chronic morphine intoxication in GABA-ergic neurons and astrocytes surrounding. In the thalamic region the activation of the degradation the neurotransmitter could arise again, due to the transfer of information within neurons after binding morphine with m-opioid receptors. It is known that long-term intake of morphine in the body causes in the target neurons activation of adenylate cyclase system, which is responsible for regulation of many intracellular processes, including inside of the mitochondrial.

The emergence of a similar effect in the cerebellum in terms of long-MWS, may indicate other mechanisms, the beginning compensatory the activation of GABA-shunt. Thus, in the later stages after morphine withdrawal after 7-day administration in thalamus and cerebellum noted persistent conservation activation of GABA catabolism. We can assume that these changes relate to the "allostatic" adaptation of GABA due to administration morphine for a long time and can be related to the preservation of drug motivation and relapse to drug use in the long cancellation period. To determine the exact molecular mechanisms of occurrence of this phenomenon requires further studies using other indicators system GABA and the citric acid cycle.

\section{Acknowledgments}

None.

\section{Conflicts of interest}

Author declares there are no conflicts of interest.

\section{Funding}

None.

\section{References}

1. Nutt DJ. Addiction: brain mechanisms and their treatment implications. Lancet. 1996;347(8993):31-36.

2. Vetulani J. Drug addiction. Part II. Neurobiology of addiction. Pol J Pharmacology . 2001;53(4):303-317.

3. Kreek MJ, Koob GF. Drug dependence: stress and dysregulation of brain reward pathways. Drug Alcohol Depend. 1998;51(1-2):23-47.

4. Bianchi C, Ferraro L, Tanganelli S, et al. 5-Hydroxytryptamine-mediated effects of nicotine on endogenous GABA efflux from guinea-pig cortical slices. Br J Pharmacol. 1995;116(6):2724-2728.

5. Hassel B, Johannessen CU, Sonnewald U, et al. Quantification of the GABA shunt and the importance of the GABA shunt versus the 2-oxoglutarate dehydrogenase pathway in GABAergic neurons. $J$ Neurochem. 1998;71(4):1511-1518.

6. Rozanov A. The successes of modern biology. 1989;103(3):375-391.

7. De Boer Th, Bruinvels J. Assay and properties of 4-aminobutyric-2oxoglutaric acid transaminase and succinic semialdehyde dehydrogenase in rat brain tissue. $J$ Neurochem. 1977;28:471-478.

8. Prokhorova I. Methods of biochemical research. The lipid and energy metabolism, Leningrad State University. 1982;188-226.

9. Constantinople A, Zhukov A, Tyurin V, et al. The experimental and Clinical Pharmacology. 1992;55(2):9-11.

10. Koob GF, Le Moal M. Drug addiction, dysregulation of reward, and allostasis. Neuropsychopharmacology. 2001;24(2):97-129. 
11. Kruk L, Pycock J. Neurotransmitters and drugs. Croom Helm, London \& Canberra. 1983;147-155.

12. Terwilliger RZ, Beitner-Johnson D, Sevarino KA, et al. A general role for adaptations in G-proteins and the cyclic AMP system in mediating the chronic actions of morphine and cocaine on neuronal function. Brain Res. 1991;548(1-2):100-110.

13. Kosten TR, George TP. The Neurobiology of opioid dependence: implications for treatment. Sci Pract Perspect. 2002;1(1):13-20.
14. Erbs E, Faget L, Ceredig RA, et al. Impact of chronic morphine on delta opioid receptor expressing neurons in the mouse hippocampus. Neuroscience. 2015

15. Howard F, Elyssa M. Understsanding opioid reward. Trends in Neurosciences. 2015;38(4):217-225.

16. Qu CL, Huo FQ, Huang FS, Tang JS. Activation of mu-opioid receptors in the ventrolateral orbital cortex inhibits the GABAergic miniature inhibitory postsynaptic currents in rats. Neurosci Lett . 2015;592:64-69. 